\title{
Cardiomyopathy in a dog with multicentric lymphoma following treatment with several anthracyclines
}

\author{
Michihito Tagawa $^{1 *}$, Genya Shimbo ${ }^{2}$, Akiko Uemura ${ }^{3}$ and Kotaro Matsumoto ${ }^{3}$ \\ ${ }^{1}$ Veterinary Medical Center, Obihiro University of Agriculture and Veterinary Medicine, Obihiro, Japan \\ ${ }^{2}$ Veterinary Teaching Hospital, Graduate School of Veterinary Medicine, Hokkaido University, Sapporo, Japan \\ ${ }^{3}$ Department of Clinical Veterinary Science, Obihiro University of Agriculture and Veterinary Medicine, Obihiro, \\ Japan
}

\begin{abstract}
Background: Canine lymphoma is one of the most frequently occurring malignant neoplasms in dogs. Anthracyclinebased chemotherapy for the treatment of canine lymphoma is very effective; however, there is not enough evidence for the development of cardiac toxicity using several anthracyclines as chemotherapeutic agents.

Case Description: An 8-year-old, castrated, mixed-breed dog was diagnosed with multicentric lymphoma and received multi-agent chemotherapy. Complete remission was achieved, but the patient had a relapse of lymphoma. After thirdline chemotherapy with epirubicin, the patient was diagnosed with dilated cardiomyopathy. The total cumulative doses of doxorubicin, mitoxantrone, and epirubicin were 125,8 , and $125 \mathrm{mg} / \mathrm{m}^{2}$, respectively. Although the patient was treated with cardiac drugs and clinically stabilized, the patient had a relapse of lymphoma and died shortly after the diagnosis of cardiomyopathy.

Conclusion: The patient was suspected to have anthracycline-induced cardiomyopathy. Further studies are required to establish prevention and management strategies for dogs receiving potentially cardiotoxic therapies, such as anthracyclines.
\end{abstract}

Keywords: AICM, Anthracycline-induced cardiomyopathy, Dog, Lymphoma.

\section{Introduction}

Canine lymphoma is one of the most frequently occurring malignant neoplasms in dogs (Zandvliet, 2016). Classification of lymphoma in dogs is based on anatomic location, and the most common anatomic form of lymphoma is the multicentric form that affects the peripheral lymph nodes. Lymphoma is the most commonly managed neoplasia in veterinary medicine, and several chemotherapeutic protocols have been developed (Zandvliet, 2016). In veterinary medicine, anthracyclines, such as doxorubicin and epirubicin, are commonly administered for a variety of malignancies, including lymphoma (Elliott et al., 2013; Curran and Thamm, 2016; Wang et al., 2016). Anthracycline-based chemotherapy for the treatment of canine lymphoma is very effective, and the increased risk of cardiotoxicity in patients using doxorubicin is very well known (Mauldin et al., 1992). However, there is not enough evidence for the development of cardiac toxicity following use of a combination of anthracyclines as chemotherapeutic agents. Here, we describe a case of multicentric lymphoma in a dog who developed dilated cardiomyopathy after treatment with several potentially cardiotoxic drugs.

\section{Case Details}

An 8-year-old, castrated, mixed-breed dog weighing $14.2 \mathrm{~kg}$ was referred to the Veterinary Medical Center,
Obihiro University of Agriculture and Veterinary Medicine, for investigation of superficial lymph node enlargement for 1 month. Based on cytological findings and clonality analysis (Lana et al., 2006), the dog was diagnosed with multicentric high-grade B-cell lymphoma, clinical stage $\mathrm{V}$ (sub-stage $\mathrm{b}$ ). The dog received 1-asparaginase $\left(5,000 \mathrm{IU} / \mathrm{m}^{2}\right.$, SC) for induction, which was complicated by tumor lysis syndrome. After recovery from tumor lysis syndrome, treatment with 25-week multi-agent chemotherapy (Garrett et al., 2002), which consisted of vincristine (0.5 $\mathrm{mg} / \mathrm{m}^{2}$, IV), cyclophosphamide $\left(250 \mathrm{mg} / \mathrm{m}^{2}\right.$, IV), doxorubicin $\left(25 \mathrm{mg} / \mathrm{m}^{2}\right.$, IV), and prednisolone ( $2 \mathrm{mg} / \mathrm{kg}, \mathrm{PO}, \mathrm{q} 24 \mathrm{~h}$ ), was administered from April to October 2018 (Fig. 1). The patient achieved complete remission and was doing well, but the lymphoma relapsed 6 months after first-line chemotherapy. Reinduction was attempted using the same protocol from April to October 2019, which replaced third and fourth doxorubicin with mitoxantrone because of the risk of cumulative toxicity of doxorubicin. The cumulative doxorubicin dose was $125 \mathrm{mg} / \mathrm{m}^{2}$. Before second doxorubicin, echocardiographic examination was performed, showing normal cardiac function with an estimated left ventricular ejection fraction (LVEF) of $68.6 \%$ and left ventricle fractional shortening (LVFS) of $37.6 \%$ (Fig. 2a). In addition, diastolic and 


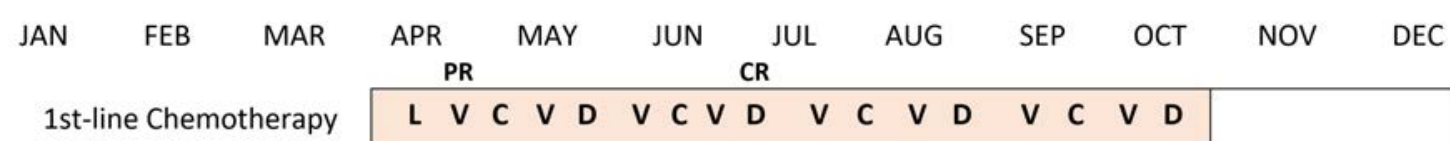

\begin{tabular}{|l|lllllllllllllllll|l|l|}
\hline R & \multicolumn{1}{l}{ CR } \\
\hline 2nd-line Chemotherapy & V & C & V & D & V & C & V & D & V & C & V & M & V & C & V & M & & \\
\hline
\end{tabular}

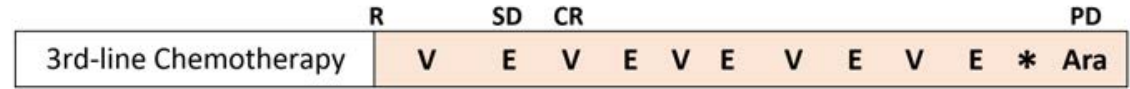

Fig. 1. Treatment schedule and clinical response of the case. $\mathrm{L}=1$-asparaginase; $\mathrm{V}=$ vincristine; $\mathrm{C}=$ cyclophosphamide; $\mathrm{D}=$ doxorubicin; $\mathrm{M}=$ mitoxantrone; $\mathrm{E}=$ epirubicin; $\mathrm{Ara}=$ cytosine arabinoside; $\mathrm{R}=$ relapse; $\mathrm{PR}=$ partial response; $\mathrm{CR}=$ complete response; $\mathrm{SD}=$ stable disease; and $\mathrm{PD}=$ progressive disease (Vail et al., 2010). The asterisk indicates the time of onset of AICM.

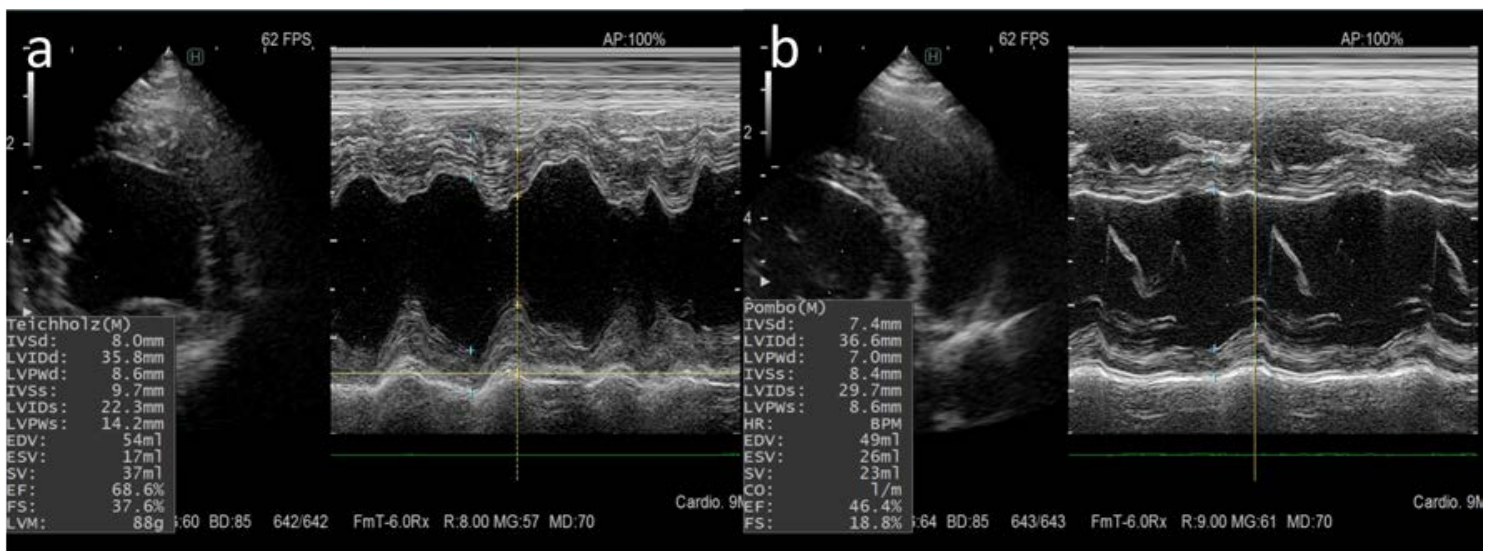

Fig. 2. M-mode echocardiograms of the case. (a): Normal cardiac function was seen in six cycles of doxorubicin administration. (b): A marked reduction in ventricular contractility was observed at 1 week after administration of six cycles of epirubicin.

systolic left ventricular wall thicknesses were 8.6 and $14.2 \mathrm{~mm}$, respectively. A mitoxantrone dose of $4 \mathrm{mg} / \mathrm{m}^{2}$ was administered twice. The dog achieved a second complete remission, but relapsed again 6 months after second-line chemotherapy. Subsequently, the dog received third-line chemotherapy, including epirubicin $\left(25 \mathrm{mg} / \mathrm{m}^{2}, \mathrm{IV}\right)$ and vincristine, resulting in high cytocidal effects. One week after five cycles of epirubicin (cumulative epirubicin dose, $125 \mathrm{mg}$ / $\mathrm{m}^{2}$ ), the dog was admitted to the hospital with a history of orthopnea, swelling of legs, and fatigue. On physical examination, muffled heart sounds were identified. Thoracic radiographs revealed generalized cardiomegaly (Fig. 3), and vertebral heart size was 11.5 (normal range, $9.7 \pm 0.5$ vertebrae). Electrocardiography (ECG) showed accelerated idioventricular rhythm with a wide QRS complex (Fig. 4). Echocardiography revealed severe diffuse hypokinesia with an LVEF of $46.4 \%$ and LVFS of $18.8 \%$ (Fig. 2b), and reduced diastolic and systolic left ventricular wall thickness (7.0 and $8.6 \mathrm{~mm}$, respectively). Troponin I was 1.185
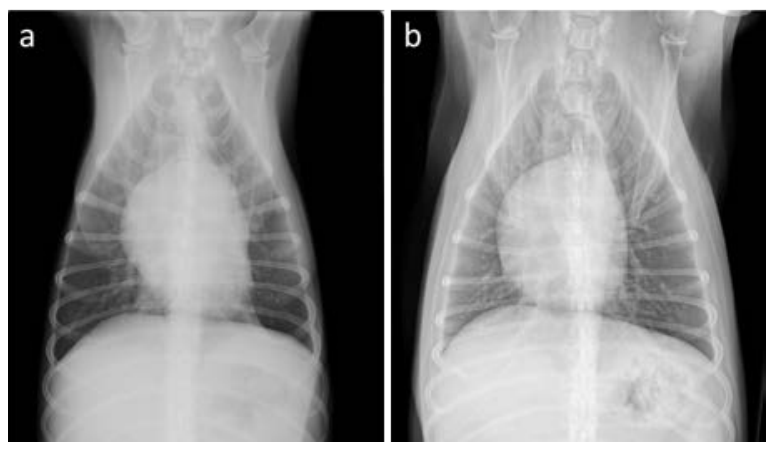

Fig. 3. Thoracic radiographs of the case. On comparing with that (a): before first doxorubicin administration, (b): generalized cardiomegaly was observed at 1 week after the administration of six cycles of epirubicin.

ng/ml (FUJIFILM VET Systems, Tokyo, reference range; $0.006-0.129 \mathrm{ng} / \mathrm{ml})$. Based on these findings, a diagnosis of anthracycline-induced cardiomyopathy (AICM) was established. The dog was treated with 


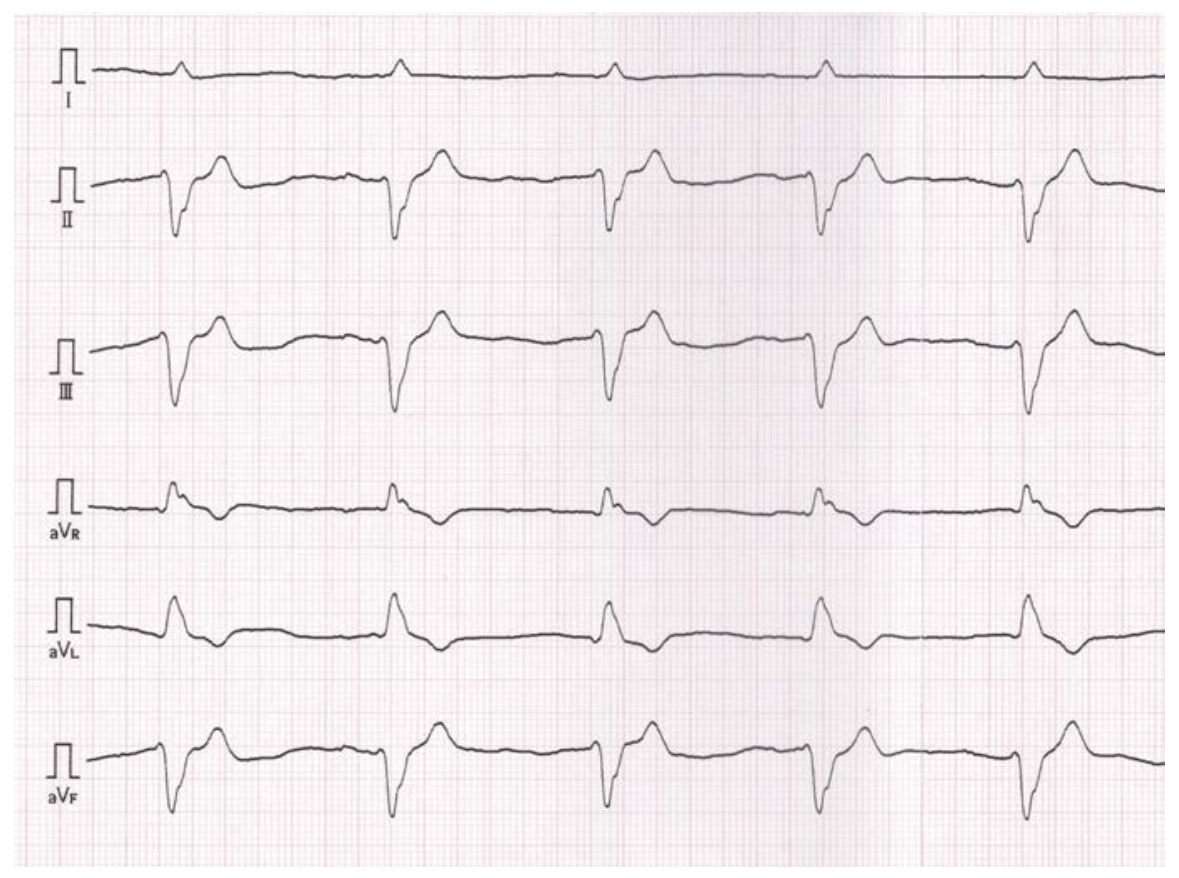

Fig. 4. Six-lead electrocardiogram, which was obtained when cardiomyopathy was found, showing accelerated idioventricular rhythm with a wide QRS complex, heart rate of 94 beats/ minutes, and cardiac axis of $-76^{\circ}(50 \mathrm{~mm} /$ seconds; $1 \mathrm{~cm}=1 \mathrm{mV})$.

benazepril $(0.32 \mathrm{mg} / \mathrm{kg}, \mathrm{PO}, \mathrm{q} 24 \mathrm{~h})$ and pimobendane $(0.16 \mathrm{mg} / \mathrm{kg}, \mathrm{PO}, \mathrm{q} 12 \mathrm{~h})$. Seven days after the treatment for cardiomyopathy, the dog was clinically stable, and echocardiography revealed improved cardiac function with LVEF of $66.2 \%$ and LVFS of $30.4 \%$. However, after 9 days, the condition worsened, and a large number of lymphoblast cells were observed in the peripheral blood smear of the dog. Cytosine arabinoside (300 mg/ $\mathrm{m}^{2}$, IV, drip infusion for 3 hours) was administered, but the dog subsequently died 22 days after the diagnosis of cardiomyopathy. Necropsy was not performed.

\section{Discussion}

Anthracyclines, such as doxorubicin and epirubicin, are highly effective and frequently used antineoplastic drugs prescribed for a variety of malignancies, especially lymphoma (Elliott et al., 2013; Curran and Thamm, 2016). However, these drugs may have side effects such as myelotoxicity, nausea, vomiting, alopecia, and tissue necrosis in cases of extravasation, and the most serious chronic effects of anthracyclines are dose-dependent and irreversible cardiotoxicity. The mechanisms associated with anthracycline-induced cardiotoxicity involve inflammation, oxidative stress, apoptosis, mitochondrial impairment, and dysregulation of autophagy, which have not been fully understood (Prathumsap et al., 2020). Anthracycline-induced cardiotoxicity is characterized by cardiomyopathy, ventricular dysfunction, pericarditis-myocarditis syndrome, or arrhythmias. Regarding doxorubicin, chronic cardiotoxicity is most commonly seen with doses that exceed $180 \mathrm{mg} / \mathrm{m}^{2}$ or more than siz doses in dogs, but cardiotoxicity can occur with any cumulative dose (Mauldin et al., 1992; Willcox et al., 2020).

Epirubicin, a stereoisomer of doxorubicin, has been reported to be less cardiotoxic than doxorubicin without compromising anti-tumor efficacy (Smith et al., 2010). Relative to doxorubicin, the equivalence ratio for epirubicin was 0.7 (McGowan et al., 2017). It is now being used instead of doxorubicin to reduce cardiotoxicity; however, the toxicity of epirubicin has not been fully evaluated in veterinary medicine, and the absolute maximum cumulative dose of epirubicin in dogs has not been defined (Kim et al., 2007; Marrington et al., 2012; Elliott et al., 2013). It has been reported that AICM occurs in dogs receiving epirubicin and doxorubicin (Lee et al., 2015). The report suggested that previous anthracycline therapy could increase the risk of cardiotoxicity. In this report, the dog was treated with cumulative epirubicin doses of $125 \mathrm{mg} / \mathrm{m}^{2}$, with previously administered six cycles of doxorubicin. Mitoxantrone, a synthetic anthracenedione, was developed as a doxorubicin analogue in a program to find a cytotoxic agent with decreased cardiotoxicity compared with doxorubicin (Alderton et al., 1992). In fact, past studies using dog models revealed that mitoxantrone was less cardiotoxic than doxorubicin, and mitoxantrone did not show cardiotoxicity in dogs that were pretreated with a threshold cardiotoxic dose of doxorubicin (Henderson et al., 1982; Sparano et al., 1982; Tham et al., 1987). However, a recent large human cohort study reported that mitoxantrone is cardiotoxic 
and four to five times more toxic than doxorubicin (Feijen et al., 2019). Further studies are required to clarify the additive or synergistic interactions of anthracyclines and other cardiotoxic drugs in dogs. In human medicine, therapeutic strategies for anthracycline-induced heart failure are not well studied. Angiotensin converting enzyme inhibitor (ACEI), diuretics, and $\beta$-blockers are used, but once symptomatic, anthracycline-induced cardiotoxicity is associated with markedly decreased survival (Cai et al., 2019). In addition, a recent study showed that administration of ACEI or a combination of betablockers at the early detection of anthracycline-induced cardiac insufficiency restored LVEF and reduced cardiac events (Mehta et al., 2018). However, poor tolerance to beta-blockade initiation in a human AICM patient has been reported (Tabet et al., 2006). In this study, the dog was treated with ACEI and a positive inotropic agent, following which the dog became clinically stable and echocardiographic improvement was observed. However, it was difficult to evaluate the efficacy of these drugs because the patient had a relapse of lymphoma and died shortly after the diagnosis of cardiomyopathy. There are four main strategies to protect against cardiotoxicity induced by anthracyclines: decreasing lifetime cumulative dose, prolonged intravenous infusion, liposomal formulation, and the addition of dexrazoxane (Graffagnino et al., 2020). Liposomal encapsulated doxorubicin and dexrazoxane administration has been reported in a few veterinary studies, but the long-term cardioprotective effects of these strategies have not been evaluated (FitzPatrick et al., 2010; Teske et al., 2011). Furthermore, these strategies are not widely used in veterinary medicine because of the short life span of dogs and the high costs incurred. In humans, LVEF is a recommended method used to evaluate the systolic function in patients treated with anthracycline drugs (Cai et al., 2019). Cancer therapeutics-related cardiac dysfunction is defined as a decrease in the LVEF by $>10 \%$ points to a value below the lower limit of normal (usually LVEF $\geq 50 \%$ ) (Zamorano et al., 2016). However, although LVFS is a recommended method for evaluating dogs receiving doxorubicin (Surachetpong et al., 2016), the diagnostic criteria for AICM of dogs have not been described, and the usefulness of LVFS in monitoring dogs treated with doxorubicin has not been evaluated well. In this case report, the diagnosis of AICM was based on echocardiography, ECG findings, and heart failure symptoms. Cardiac biomarkers, such as troponin, can be good indicators of doxorubicin-induced myocardial injury and can provide useful diagnostic information, especially when used in combination with echocardiography assessment (Octavia et al., 2012). In veterinary medicine, it was reported that dogs treated with doxorubicin had increased troponin levels, which were detected before echocardiographic value changes (Surachetpong et al., 2016). Furthermore,
ECG abnormalities could occur after dogs received a low doxorubicin dosage (Mauldin et al., 1992). It is necessary to establish a monitoring strategy for the early detection of cardiotoxic effects in dogs if the maximum cumulative dose of doxorubicin is achieved or several cardiotoxic agents are used.

In this case report, we described suspected AICM in a dog receiving several cardiotoxic drugs, including doxorubicin, epirubicin, and mitoxantrone. The additive or synergistic effect of these cardiotoxic drugs in dogs is poorly documented. Further studies are required to establish prevention and management strategies for dogs receiving potentially cardiotoxic therapies such as anthracyclines.

\section{Conflict of interest}

The authors declare that there is no conflict of interest.

\section{References}

Alderton, P.M., Gross, J. and Green, M.D. 1992. Comparative study of doxorubicin, mitoxantrone, and epirubicin in combination with ICRF-187 (ADR-529) in a chronic cardiotoxicity animal model. Cancer Res. 52, 194-201.

Cai, F., Luis, M.A.F., Lin, X., Wang, M., Cai, L., Cen, C. and Biskup, E. 2019. Anthracycline-induced cardiotoxicity in the chemotherapy treatment of breast cancer: preventive strategies and treatment. Mol. Clin. Oncol. 11, 15-23.

Curran, K. and Thamm, D.H. 2016. Retrospective analysis for treatment of naïve canine multicentric lymphoma with a 15-week, maintenance-free CHOP protocol. Vet. Comp. Oncol. 14, 147-155.

Elliott, J.W., Cripps, P., Marrington, A.M., Grant, I.A. and Blackwood, L. 2013. Epirubicin as part of a multi-agent chemotherapy protocol for canine lymphoma. Vet. Comp. Oncol. 11, 185-198.

Feijen, E.A.M., Leisenring, W.M., Stratton, K.L., Ness, K.K., van der Pal, H.J.H., van Dalen, E.C., Armstrong, G.T., Aune, G.J., Green, D.M., Hudson, M.M., Loonen, J., Oeffinger, K.C., Robison, L.L., Yasui, Y., Kremer, L.C.M. and Chow, E.J. 2019. Derivation of anthracycline and anthraquinone equivalence ratios to Doxorubicin for Late-Onset Cardiotoxicity. JAMA Oncol. 5, 864-871.

FitzPatrick, W.M., Dervisis, N.G. and Kitchell, B.E. 2010. Safety of concurrent administration of dexrazoxane and doxorubicin in the canine cancer patient. Vet. Comp. Oncol. 8, 273-282.

Garrett, L.D., Thamm, D.H., Chun, R., Dudley, R. and Vail, D.M. 2002. Evaluation of a 6-month chemotherapy protocol with no maintenance therapy for dogs with lymphoma. J. Vet. Intern. Med. 16, 704-709.

Graffagnino, J., Kondapalli, L., Arora, G., Hawi, R. and Lenneman, C.G. 2020. Strategies to prevent cardiotoxicity. Curr. Treat. Options Oncol. 21, 32.

Henderson, B.M., Dougherty, W.J., James, V.C., Tilley, L.P. and Noble, J.F. 1982. Safety assessment of a 
new anticancer compound, mitoxantrone, in beagle dogs: comparison with doxorubicin. I. Clinical observations. Cancer Treat. Rep. 66, 1139-1143.

Kim, S.E., Liptak, J.M., Gall, T.T., Monteith, G.J. and Woods, J.P. 2007. Epirubicin in the adjuvant treatment of splenic hemangiosarcoma in dogs: 59 cases (1997-2004). J. Am. Vet. Med. Assoc. 231, $1550-1557$.

Lana, S.E., Jackson, T.L., Burnett, R.C., Morley, P.S. and Avery, A.C. 2006. Utility of polymerase chain reaction for analysis of antigen receptor rearrangement in staging and predicting prognosis in dogs with lymphoma. J. Vet. Intern. Med. 20, 329-334.

Lee, Y.R. Kang, M.H. and Park, H.M. 2015. Anthracycline-induced cardiomyopathy in a dog treated with epirubicin. Can. Vet. J. 56, 571-574.

Marrington, A.M., Killick, D.R., Grant, I.A. and Blackwood, L. 2012. Toxicity associated with epirubicin treatments in a large case series of dogs. Vet. Comp. Oncol. 10, 113-123.

Mauldin, G.E., Fox, P.R., Patnaik, A.K., Bond, B.R., Mooney, S.C. and Matus, R.E. 1992. Doxorubicininduced cardiotoxicosis. Clinical features in 32 dogs. J. Vet. Intern. Med. 6, 82-88.

McGowan, J.V., Chung, R., Maulik, A., Piotrowska, I., Walker, J.M. and Yellon, D.M. 2017. Anthracycline chemotherapy and cardiotoxicity. Cardiovasc. Drugs. Ther. 31, 63-75.

Mehta, L.S., Watson, K.E., Barac, A., Beckie, T.M., Bittner, V., Cruz-Flores, S., Dent, S., Kondapalli, L., Ky, B., Okwuosa, T., Piña, I.L., Volgman, A.S. and American Heart Association Cardiovascular Disease in Women and Special Populations Committee of the Council on Clinical Cardiology, Council on Cardiovascular and Stroke Nursing; and Council on Quality of Care and Outcomes Research. 2018. Cardiovascular disease and breast cancer: where these entities intersect: a scientific statement from the American heart association. Circulation 137, e30-e66.

Octavia, Y., Tocchetti, C.G, Gabrielson, K.L., Janssens, S., Crijns, H.J. and Moens, A.L. 2012. Doxorubicininduced cardiomyopathy: from molecular mechanisms to therapeutic strategies. J. Mol. Cell Cardiol. 52, 1213-1225.

Prathumsap, N., Shinlapawittayatorn, K., Chattipakorn, S.C. and Chattipakorn, N. 2020. Effects of doxorubicin on the heart: From molecular mechanisms to intervention strategies. Eur. J. Pharmacol. 866, 172818.

Smith, L.A., Cornelius, V.R., Plummer, C.J., Levitt, G., Verrill, M., Canney, P. and Jones, A. 2010. Cardiotoxicity of anthracycline agents for the treatment of cancer: systematic review and metaanalysis of randomised controlled trials. BMC Cancer 10, 337.
Sparano, B.M., Gordon, G., Hall, C., Iatropoulos, M.J. and Noble, J.F. 1982. Safety assessment of new anticancer compound, mitoxantrone, in beagle dogs: comparison with doxorubicin. II. Histologic and ultrastructural pathology. Cancer Treat. Rep. $66,1145-1158$.

Surachetpong, S.D., Teewasutrakul, P. and Rungsipipat, A. 2016. Serial measurements of cardiac troponin I (cTnI) in dogs treated with doxorubicin. Jpn. J. Vet. Res. 64, 221-233.

Tabet, J.Y., Meurin, P., Ben Driss, A., Berthaux, X., Weber, H., Renaud, N. and Cohen Solal, A. 2006. Beta-blockade intolerance in anthracycline-induced cardiomyopathy. Int. J. Cardiol. 106, 132-134.

Teske, E., Rutteman, G. R., Kirpenstein, J. and Hirschberger, J. 2011. A randomized controlled study into the efficacy and toxicity of pegylated liposome encapsulated doxorubicin as an adjuvant therapy in dogs with splenic haemangiosarcoma. Vet. Comp. Oncol. 9, 283-9.

Tham, P., Dougherty, W., Iatropoulos, M.J., Gordon, G., James, V.C., Hall, C. and Noble, J.F. 1987. The effect of mitoxantrone treatment in beagle dogs previously treated with minimally cardiotoxic doses of doxorubicin. Am. J. Pathol. 128, 121-130.

Vail, D.M., Michels, G.M., Khanna, C., Selting, K.A. and London, C.A., Veterinary Cooperative Oncology Group. 2010. Response evaluation criteria for peripheral nodal lymphoma in dogs (v1.0)--a Veterinary Cooperative Oncology Group (VCOG) consensus document. Vet. Comp. Oncol. 8, 28-37.

Wang, S.L., Lee, J.J. and Liao, A.T. 2016. Comparison of efficacy and toxicity of doxorubicin and mitoxantrone in combination chemotherapy for canine lymphoma. Can. Vet. J. 57, 271-276.

Willcox, J.L., Belanger, C., Burton, J.H., Yu, L., Ueda, Y., Visser, L.C., Skorupski, K. and Stern, J.A. 2020. Intramuscular diphenhydramine does not affect acute doxorubicin infusion-related arrhythmia number or severity in a prospective crossover study in canine lymphoma: a pilot study. Front. Vet. Sci. 7,368 .

Zamorano, J.L., Lancellotti, P., Rodriguez Muñoz, D., Aboyans, V., Asteggiano, R., Galderisi, M., Habib, G., Lenihan, D.J., Lip, G.Y.H., Lyon, A.R., Lopez Fernandez, T., Mohty, D., Piepoli, M.F., Tamargo, J., Torbicki, A. and Suter, T.M., ESC Scientific Document Group. 2016. ESC Position paper on cancer treatments and cardiovascular toxicity developed under the auspices of the ESC Committee for Practice Guidelines: the task force for cancer treatments and cardiovascular toxicity of the European Society of Cardiology (ESC). Eur. Heart J. 37, 2768-2801.

Zandvliet, M. 2016. Canine lymphoma: a review. Vet. Q. 36, 76-104. 